\title{
Poisson Exponential Power Distribution: Properties and Application
}

\author{
Ramesh Kumar Joshi ${ }^{1}$, Vijay Kumar ${ }^{2}$ \\ ${ }^{1}$ Trichandra Multiple Campus, Saraswoti Sadan, Kathmandu, Nepal \\ ${ }^{2}$ Department of Mathematics and Statistics, DDU Gorakhpur University, Gorakhpur, India
}

\begin{tabular}{ll}
\hline ARTICLE INFO & ABSTRACT \\
\hline $\begin{array}{l}\text { Published Online: } \\
30 \text { November 2020 }\end{array}$ & $\begin{array}{l}\text { In this study, we have established a new three-parameter Poisson Exponential Power distribution } \\
\text { using the Poisson-G family of distribution. We have presented the mathematical and statistical } \\
\text { properties of the proposed distribution including probability density function, cumulative } \\
\text { distribution function, reliability function, hazard rate function, quantile, the measure of skewness, } \\
\text { and kurtosis. The parameters of the new distribution are estimated using the maximum likelihood } \\
\text { estimation (MLE) method, and constructed the asymptotic confidence intervals also the Fisher } \\
\text { information matrix is derived analytically to obtain the variance-covariance matrix for MLEs. All } \\
\text { the computations are performed in R software. The potentiality of the proposed distribution is } \\
\text { revealed by using some graphical methods and statistical tests taking a real dataset. We have } \\
\text { empirically proven that the proposed distribution provided a better fit and more flexible in } \\
\text { comparison with some other lifetime distributions. }\end{array}$ \\
Corresponding Author:
\end{tabular}

KEYWORDS: Poisson distribution, Exponential, Reliability, Hazard rate.

\section{INTRODUCTION}

The exponential distribution is the most frequently used distribution due to the existence of simple elegant closedform solutions to many survival analysis problems. The failure rate of the exponential distribution is constant but in real practice, the failure rates are not always constant. Hence in some situations, it seems to be inadequacy and unrealistic. For this, some modifications are desirable to make exponential distribution more flexible. In recent, a new class of models has been introduced based on the adjustment of exponential distribution.

Gupta and Kundu (1999) introduced the generalized exponential (GE) distribution, this extended family can accommodate data with increasing and decreasing failure rate functions, Nadarajah and Kotz (2006) have introduced a generalization referred to as the beta exponential distribution generated from the logit of a beta random variable. There are lots of lifetime models which are obtained by compounding with Zero truncated Poisson distribution some of them are as follows,

Kus (2007) has introduced the two-parameter exponential Poisson (EP) distribution by compounding exponential distribution with zero truncated Poisson distribution with a decreasing failure rate. The CDF of PE distribution is,

$$
F(t ; \beta, \lambda)=\frac{1}{\left(1-e^{-\lambda}\right)}\left[1-e^{\left\{-\lambda\left(1-e^{-\beta t}\right)\right\}}\right] ; t>0,(\beta, \lambda)>0
$$

While Barreto-Souza and Cribari-Neto (2009) have introduced generalized EP distribution having the decreasing or increasing or upside-down bathtub shaped failure rate. This is the generalization of the distribution proposed by Kus (2007) adding a power parameter to this distribution.

Following a similar approach, Percontini et al. (2013) have proposed the five-parameter beta Weibull Poisson distribution, which is obtained by compounding the Weibull Poisson and beta distributions. Following the same trend, Cancho (2011) has developed a new distribution family also based on the exponential distribution with an increasing failure rate function known as Poisson exponential (PE) distribution. The cumulative distribution function of PE distribution can be expressed as

$$
F(y ; \lambda, \theta)=1-\frac{1-\mathrm{e}^{\left\{-\theta\left(1-e^{-\lambda y}\right)\right\}}}{\left(1-e^{-\lambda}\right)} ; y>0,(\lambda, \theta)>0
$$

A two-parameter Poisson-exponential with increasing failure rate has been defined by (Louzada-Neto et al., 2011) 
by using the same approach as used by (Cancho, 2011) under the Bayesian approach. Alkarni and Oraby (2012) have introduced a new lifetime family of distribution with a decreasing failure rate which is obtained by compounding truncated Poisson distribution and a lifetime model. The cumulative distribution function of the Poisson generating family is given by,

$$
F_{P}(x ; \lambda, \underline{\delta})=1-\frac{1-e^{\{-\lambda G(y, \underline{\delta})\}}}{\left(1-e^{-\lambda}\right)} ; \lambda>0
$$

Where $\underline{\delta}$ the parameter is space and $G(y, \underline{\delta})$ is the cumulative distribution function of any distribution. Using a parallel approach the Weibull power series class of distributions with Poisson has presented by (Morais \& Barreto-Souza, 2011). Mahmoudi and Sepahdar (2013) have defined a new four-parameter distribution with increasing, decreasing, bathtub-shaped, and unimodal failure rate called as the exponentiated Weibull-Poisson (EWP) distribution which has obtained by compounding exponentiated Weibull (EW) and Poisson distributions. Similarly, Lu and Shi (2012) have created the new compounding distribution named the Weibull-Poisson distribution having the shape of decreasing, increasing, upside-down bathtub-shaped, or unimodal failure rate function. Furthur Kaviayarasu and Fawaz (2017) have made an extensive study on WeibullPoisson distribution through a reliability sampling plan. Kyurkchiev et al. (2018) has used the exponentiated exponential-Poisson as the software reliability model. Joshi \& Kumar (2020) has developed Lindley exponential power distribution having variety of shape of failure rate function. Louzada et al. (2020) has used different estimation methods to estimate the parameter of exponential-Poisson distribution using rainfall and aircraft data.

In this study, we propose a new distribution based on the exponential power distribution has introduced by (Srivastava \& Kumar, 2011) to analyze the software reliability data having the shape of decreasing, increasing, $j$ shaped, and bathtub-shaped failure rate function for different values of the parameters. The CDF and PDF of the exponential power distribution are respectively as

$$
G(x)=1-\exp \left[1-e^{\beta x^{\alpha}}\right] ; \alpha, \beta>0, x>0
$$

And

$$
g(x)=\alpha \beta x^{\alpha-1} e^{\beta x^{\alpha}} \exp \left[1-e^{\beta x^{\alpha}}\right] ; \alpha, \beta>0, x>0
$$

The different sections of this study are arranged as follows; in Section 2 we present the new distribution Poisson exponential power (PEP) with its mathematical and statistical properties. We comprehensively discuss the maximum likelihood estimation method in Section 3. In Section 4 using a real dataset, we present the estimated values of the model parameters and their corresponding asymptotic confidence intervals and fisher information matrix. Besides, we have illustrated the different test criteria to assess the goodness of fit of the proposed model. Some concluding remarks are presented in Section 5.

\section{THE POISSON EXPONENTIAL POWER (PEP) DISTRIBUTION}

Alkarni and Oraby (2012) have introduced a new lifetime class with a decreasing failure rate which is obtained by compounding truncated Poisson distribution and a lifetime distribution, where the compounding procedure follows the same way that was previously carried out by (Adamidis \& Loukas, 1998). Let $G(x)$ and $g(x)$ be the baseline cumulative distribution function and probability density function respectively then the Poisson family with $\mathrm{CDF}$ and PDF may be expressed as,

$$
\begin{aligned}
& F(x)=1-\frac{1}{\left(1-e^{-\lambda}\right)}[1-\exp \{-\lambda(1-G(x))\}] ; x>0, \lambda>0 \\
& f(x)=\frac{1}{\left(1-e^{-\lambda}\right)} \lambda g(x) \exp \{-\lambda(1-G(x))\} \quad ; x>0, \lambda>0
\end{aligned}
$$

Substituting (4) and (5) in (6) and (7) then the Poisson exponential power distribution can be defined as, Let $\mathrm{X}$ be a nonnegative random variable representing the survival time of an item or component or a system of some population. The random variable $\mathrm{X}$ is said to follow the PEP distribution with parameters $(\alpha, \beta, \lambda)>0$ if its cumulative distribution function is given by

$$
F(x ; \alpha, \beta, \lambda)=1-\frac{1}{\left(1-e^{-\lambda}\right)}\left[1-\exp \left\{-\lambda \exp \left(1-e^{\beta x^{\alpha}}\right)\right\} ; x>0,\right.
$$

And its corresponding probability density function is

$$
f(x ; \alpha, \beta, \lambda)=\frac{\alpha \beta \lambda}{\left(1-e^{-\lambda}\right)} x^{\alpha-1} \exp \left(1+\beta x^{\alpha}-e^{\beta x^{\alpha}}\right) \exp \left\{-\lambda \exp \left(1-e^{\beta x^{\alpha}}\right)\right\} ; x>0
$$

\section{Reliability function:}

The reliability function $R(t)$, which is the probability of an item not failing up to time $t$, is defined by $R(t)=1-F(t)$. The survival /reliability function of the Poisson exponential power distribution is given by

$$
R(t ; \alpha, \beta, \lambda)=\frac{1}{\left(1-e^{-\lambda}\right)}\left[1-\exp \left\{-\lambda \exp \left(1-e^{\beta t^{\alpha}}\right)\right\}\right] ; t>0
$$

\section{The hazard rate function (HRF)}

The hazard rate function for the PEP distribution can be defined as,

$$
h(x)=\frac{f(x)}{R(x)}=\frac{f(x)}{1-F(x)} ; 0<x<\infty
$$

where $\mathrm{R}(\mathrm{x})$ is a reliability function.

Hence let, $\mathrm{X} \sim \operatorname{PEP}(\alpha, \beta, \lambda)$ then its hazard rate function is

$$
h(x ; \alpha, \beta, \lambda)=\alpha \beta \lambda \frac{x^{\alpha-1} \exp \left(1+\beta x^{\alpha}-e^{\beta x^{\alpha}}\right)}{\exp \left\{\lambda \exp \left(1-e^{\beta x^{\alpha}}\right)\right\}-1} ; x>0
$$




\section{"Poisson Exponential Power Distribution: Properties and Application"}

In Figure 1 we have presented the graph for PDF and hazard function for PEP distribution for different values of the parameters. From Figure 1 (left panel), the density function of the PEP distribution can bear different shapes according to the values of the parameters. Figure 1 (right panel) demonstrates the increasing, decreasing, the $\mathrm{j}$-shaped, and constant shape of the hazard rate.
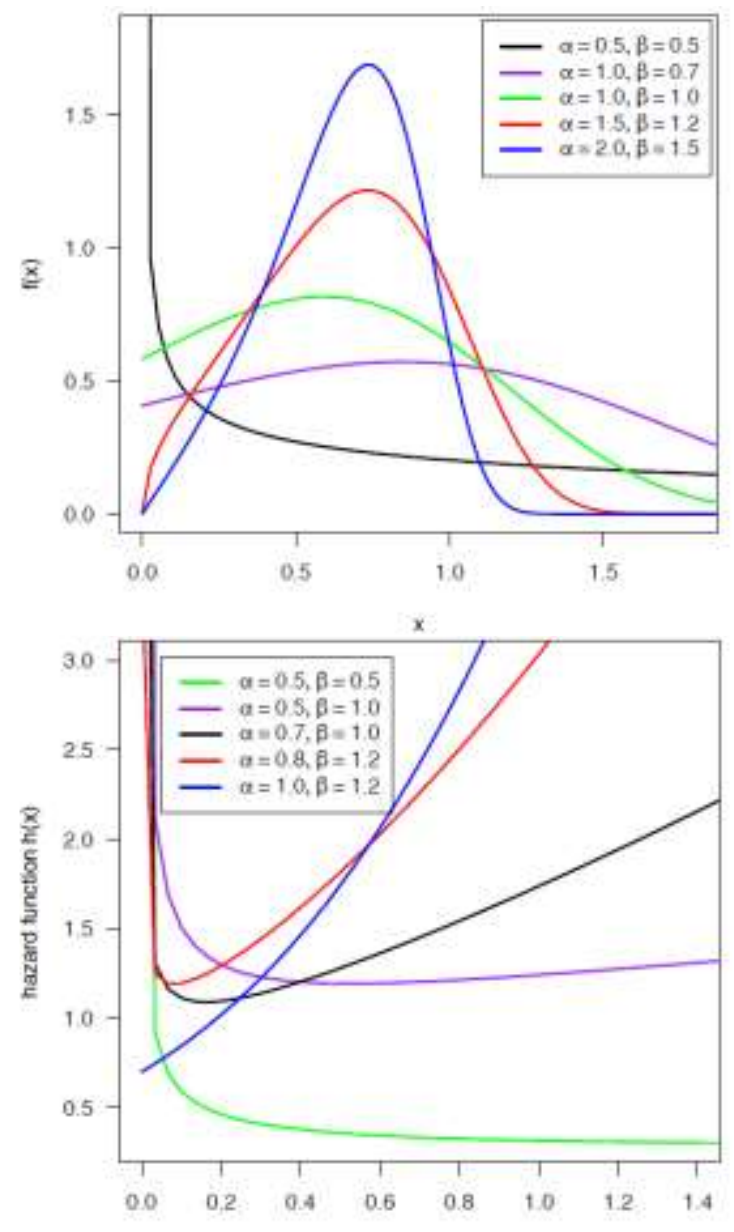

Figure 1. Graph of PDF (upper panel) and hazard function (lower panel) for different values of the parameters.

\section{The quantile function of PEP distribution}

According to Hyndman and Fan (1996), the value of the $\mathrm{p}^{\text {th }}$ quantile can be obtained by solving the following equation,

$$
Q(p)=F^{-1}(p)
$$

And we get the quantile function by inverting CDF of PEP as

$$
Q(p)=\left[\frac{1}{\beta} \ln \left[1-\left[\ln \left\{-\frac{1}{\lambda} \ln \left\{1-(1-p)\left(1-e^{-\lambda}\right)\right\}\right\}\right]\right]\right]^{\frac{1}{\alpha}} ; 0<p<1
$$

For the generation of the random numbers of the PEP distribution, we suppose simulating values of random variable $\mathrm{X}$ with the $\mathrm{CDF}$ (8). Let $\mathrm{U}$ denote a uniform random variable in $(0,1)$, then the simulated values of $X$ can be obtained by

$$
x=\left[\frac{1}{\beta} \ln \left[1-\left[\ln \left\{-\frac{1}{\lambda} \ln \left\{1-(1-u)\left(1-e^{-\lambda}\right)\right\}\right\}\right]\right]\right]^{\frac{1}{\alpha}} ; 0<u<1
$$

\section{Median of PEP distribution}

The median of $X$ from the PEP distribution is simply obtained by replacing $p=0.5$ in equation (12) which gives

$$
\text { Median }=\left[\frac{1}{\beta} \ln \left[1-\left[\ln \left\{-\frac{1}{\lambda} \ln \left\{1-0.5\left(1-e^{-\lambda}\right)\right\}\right\}\right]\right]\right]^{\frac{1}{\alpha}}
$$

Skewness and Kurtosis:

The Bowley's measure of skewness based on quartiles was defined by (Kennedy \& Keeping, 1962) as,

$$
S_{k}(B)=\frac{Q(3 / 4)+Q(1 / 4)-2 Q(1 / 2)}{Q(3 / 4)-Q(1 / 4)},
$$

and the coefficient of Moor's kurtosis measures based on octiles was defined by (Moors, 1988) is given by

$$
K_{u}(M)=\frac{Q(0.875)-Q(0.625)+Q(0.375)-Q(0.125)}{Q(3 / 4)-Q(1 / 4)},
$$

\section{MAXIMUM LIKELIHOOD ESTIMATION}

Let $X_{1}, X_{2}, \ldots, X_{n}$ be a sample of size 'n' independently and identically distributed random variables from the PEP with unknown parameters, $\alpha, \beta$ and $\lambda$ defined previously.

The likelihood function of the PEP using the PDF in equation (9) is given by:

$$
L(\alpha, \beta, \lambda \mid \underline{x})=\frac{\alpha \beta \lambda}{\left(1-e^{-\lambda}\right)} \prod_{i=1}^{n} x_{i}^{\alpha-1} \exp \left(1+\beta x_{i}^{\alpha}-e^{\beta x_{i}^{\alpha}}\right) \exp \left\{-\lambda \exp \left(1-e^{\beta x_{i}^{\alpha}}\right)\right\}
$$

It is easy to deals with natural logarithm, hence let $l(\alpha, \beta, \lambda)$ be $\log$-likelihood function,

$$
\begin{aligned}
l(\alpha, \beta, \lambda)=n+n \ln (\alpha \beta)+n \ln ( & \left.\frac{\lambda}{1-e^{-\lambda}}\right)+(\alpha-1) \sum_{i=1}^{n} \ln x_{i} \\
& \quad+\beta \sum_{i=1}^{n} x_{i}^{\alpha}-\sum_{i=1}^{n} e^{\beta x_{i}^{\alpha}}-\lambda \sum_{i=1}^{n} \exp \left(1-e^{\beta x_{i}^{\alpha}}\right)
\end{aligned}
$$

To estimate the unknown parameters of the $\operatorname{PEP}(\alpha, \beta, \lambda)$, we have to solve the following nonlinear equations equating to zero.

$$
\begin{aligned}
& \frac{\partial l}{\partial \alpha}=\frac{n}{\alpha}+\sum_{i=1}^{n} \ln x_{i}+\beta \sum_{i=1}^{n} x_{i}^{\alpha} \ln x_{i}-\beta \sum_{i=1}^{n} x_{i}^{\alpha} e^{\beta x_{i}^{\alpha}} \ln x_{i}+\beta \lambda \sum_{i=1}^{n} x_{i}^{\alpha} e^{\beta x_{i}^{\alpha}} \exp \left(1-e^{\beta x_{i}^{\alpha}}\right) \ln x_{i} \\
& \frac{\partial l}{\partial \beta}=\frac{n}{\beta}+\sum_{i=1}^{n} x_{i}^{\alpha}-\sum_{i=1}^{n} x_{i}^{\alpha} e^{\beta x_{i}^{\alpha}}+\lambda \sum_{i=1}^{n} x_{i}^{\alpha} e^{\beta x_{i}^{\alpha}} \exp \left(1-e^{\beta x_{i}^{\alpha}}\right) \\
& \frac{\partial l}{\partial \lambda}=\frac{n\left(e^{\lambda}-\lambda-1\right)}{\lambda\left(e^{\lambda}-1\right)}-\sum_{i=1}^{n} \exp \left(1-e^{\beta x_{i}^{\alpha}}\right)
\end{aligned}
$$

it is difficult to solve the above non-linear equation manually, so by using the computer software R, Mathematica, Matlab, or any other suitable programs and Newton-Raphson's iteration method one can solve these equations. Let us denote the parameter vector by $\underline{\tau}=(\alpha, \beta, \lambda)$ and the corresponding MLE of $\underline{\tau}$ as $\underline{\hat{\tau}}=(\hat{\alpha}, \hat{\beta}, \hat{\lambda})$, then the asymptotic normality results in, $(\underline{\hat{\tau}}-\hat{\tau}) \rightarrow N_{3}\left[0,(I(\underline{\tau}))^{-1}\right]$ where $I(\underline{\tau})$ is the Fisher's information matrix given by, 


$$
I(\underline{\tau})=-\left(\begin{array}{ccc}
E\left(\frac{\partial^{2} l}{\partial \alpha^{2}}\right) & E\left(\frac{\partial^{2} l}{\partial \alpha \partial \beta}\right) & E\left(\frac{\partial^{2} l}{\partial \alpha \partial \lambda}\right) \\
E\left(\frac{\partial^{2} l}{\partial \alpha \partial \beta}\right) & E\left(\frac{\partial^{2} l}{\partial \lambda^{2}}\right) & E\left(\frac{\partial^{2} l}{\partial \lambda \partial \beta}\right) \\
E\left(\frac{\partial^{2} l}{\partial \alpha \partial \lambda}\right) & E\left(\frac{\partial^{2} l}{\partial \lambda \partial \beta}\right) & E\left(\frac{\partial^{2} l}{\partial \lambda^{2}}\right)
\end{array}\right)
$$

Further differentiating (16) we get,

$\frac{\partial^{2} l}{\partial \alpha^{2}}=-\frac{n}{\alpha^{2}}+\beta \sum_{i=1}^{n} x_{i}^{\alpha}\left(\ln x_{i}\right)^{2}-\beta \sum_{i=1}^{n} x_{i}^{\alpha} e^{\beta x_{i}^{\alpha}}\left(\ln x_{i}\right)^{2}\left(1+\beta x_{i}^{\alpha}\right)$

$$
-\beta \lambda \sum_{i=1}^{n} x_{i}^{\alpha}\left(\beta x_{i}^{\alpha} e^{\beta x_{i}^{\alpha}}-\beta x_{i}^{\alpha}-1\right) \exp \left(1+\beta x_{i}^{\alpha}-e^{\beta x_{i}^{\alpha}}\right)\left(\ln x_{i}\right)^{2}
$$

$\frac{\partial^{2} l}{\partial \beta^{2}}=-\frac{n}{\beta^{2}}-\sum_{i=1}^{n} x_{i}^{2 \alpha} e^{\beta x_{i}^{\alpha}}+\lambda \sum_{i=1}^{n} x_{i}^{3 \alpha} e^{\beta x_{i}^{\alpha}} \exp \left(1-e^{\beta x_{i}^{\alpha}}\right)$

$\frac{\partial^{2} l}{\partial \lambda^{2}}=-\frac{n\left\{e^{2 \lambda}-\left(\lambda^{2}+2\right) e^{\lambda}+1\right\}}{\lambda^{2}\left(e^{\lambda}-1\right)^{2}}$

$\frac{\partial^{2} l}{\partial \alpha \partial \beta}=\sum_{i=1}^{n} x_{i}^{\alpha} \ln x_{i}-\sum_{i=1}^{n} x_{i}^{\alpha} e^{\beta \beta_{i}^{\alpha}}\left(1+\beta x_{i}^{\alpha}\right) \ln x_{i}-\lambda \sum_{i=1}^{n} x_{i}^{\alpha}\left(\beta x_{i}^{\alpha} e^{\beta \beta_{i}^{\alpha}}-\beta x_{i}^{\alpha}-1\right) \exp \left(1+\beta x_{i}^{\alpha}-e^{\beta \beta_{i}^{\alpha}}\right) \ln x_{i}$

$\frac{\partial^{2} l}{\partial \alpha \partial \lambda}=\beta \sum_{i=1}^{n} x_{i}^{\alpha} e^{\beta x_{i}^{\alpha}} \exp \left(1-e^{\beta x_{i}^{\alpha}}\right) \ln x_{i}$

$\frac{\partial^{2} l}{\partial \beta \partial \lambda}=\sum_{i=1}^{n} x_{i}^{\alpha} \exp \left(1+\beta x_{i}^{\alpha}-e^{\beta x_{i}^{\alpha}}\right)$

In practice, it is useless that the MLE has asymptotic variance $(I(\underline{\tau}))^{-1}$ because we don't know $\underline{\tau}$. Hence we approximate the asymptotic variance by plugging in the estimated value of the parameters. The common procedure is to use the observed Fisher information matrix $\Psi(\underline{\hat{\tau}})$ as an estimate of the information matrix $I(\underline{\tau})$ given by

$\Psi(\underline{\hat{\tau}})=-\left(\left.\begin{array}{ccc}\frac{\partial^{2} l}{\partial \alpha^{2}} & \frac{\partial^{2} l}{\partial \alpha \partial \beta} & \frac{\partial^{2} l}{\partial \alpha \partial \lambda} \\ \frac{\partial^{2} l}{\partial \alpha \partial \beta} & \frac{\partial^{2} l}{\partial \beta^{2}} & \frac{\partial^{2} l}{\partial \lambda \partial \beta} \\ \frac{\partial^{2} l}{\partial \alpha \partial \lambda} & \frac{\partial^{2} l}{\partial \lambda \partial \beta} & \frac{\partial^{2} l}{\partial \lambda^{2}}\end{array}\right|_{(\hat{\alpha}, \hat{\beta}, \hat{\lambda})}=-\left.H(\underline{\tau})\right|_{(\underline{\tau}=\underline{\hat{\tau}})}\right.$

where $\mathrm{H}$ is the Hessian matrix.

The Newton-Raphson algorithm to maximize the likelihood produces the observed information matrix. Therefore, the variance-covariance matrix is given by,

$\left[-H\left(\left.\underline{\tau}\right|_{(\underline{\tau}=\underline{\hat{\tau}})}\right]^{-1}=\left(\begin{array}{ccc}\operatorname{var}(\hat{\alpha}) & \operatorname{cov}(\hat{\alpha}, \hat{\beta}) & \operatorname{cov}(\hat{\alpha}, \hat{\lambda}) \\ \operatorname{cov}(\hat{\alpha}, \hat{\beta}) & \operatorname{var}(\hat{\beta}) & \operatorname{cov}(\hat{\beta}, \hat{\lambda}) \\ \operatorname{cov}(\hat{\alpha}, \hat{\lambda}) & \operatorname{cov}(\hat{\beta}, \hat{\lambda}) & \operatorname{var}(\hat{\lambda})\end{array}\right)\right.$

Hence from the asymptotic normality of MLEs, approximate $100(1-\alpha) \%$ confidence intervals for $\alpha, \beta$ and $\lambda$ can be constructed as,

$$
\hat{\alpha} \pm Z_{\alpha / 2} \sqrt{\operatorname{var}(\hat{\alpha})} \quad, \hat{\beta} \pm Z_{\alpha / 2} \sqrt{\operatorname{var}(\hat{\beta})} \quad \text { and }
$$

$\hat{\lambda} \pm Z_{\alpha / 2} \sqrt{\operatorname{var}(\hat{\lambda})}$ where $Z_{\alpha / 2}$ is the upper percentile of standard normal variate.

\section{APPLICATION WITH A REAL DATASET}

In this section, we illustrate the applicability of the PEP model using a real dataset used by former researchers. We have taken 100 observations on breaking the stress of carbon fibers (in Gba) used by (Nichols \& Padgett, 2006).

$3.70,2.74,2.73,2.50,3.60,3.11,3.27,2.87,1.47,3.11$, $4.42,2.41,3.19,3.22,1.69,3.28,3.09,1.87,3.15,4.90$, $3.75,2.43,2.95,2.97,3.39,2.96,2.53,2.67,2.93,3.22$, $3.39,2.81,4.20,3.33,2.55,3.31,3.31,2.85,2.56,3.56$, $3.15,2.35,2.55,2.59,2.38,2.81,2.77,2.17,2.83,1.92$, $1.41,3.68,2.97,1.36,0.98,2.76,4.91,3.68,1.84,1.59$, $3.19,1.57,0.81,5.56,1.73,1.59,2.00,1.22,1.12,1.71$, $2.17,1.17,5.08,2.48,1.18,3.51,2.17,1.69,1.25,4.38$, $1.84,0.39,3.68,2.48,0.85,1.61,2.79,4.70,2.03,1.80$, $1.57,1.08,2.03,1.61,2.12,1.89,2.88,2.82,2.05,3.65$

The plots of profile log-likelihood function for the parameters $\alpha, \beta$, and $\lambda$ have been displayed in Figure 2 and noticed that the ML estimates can be uniquely determined.
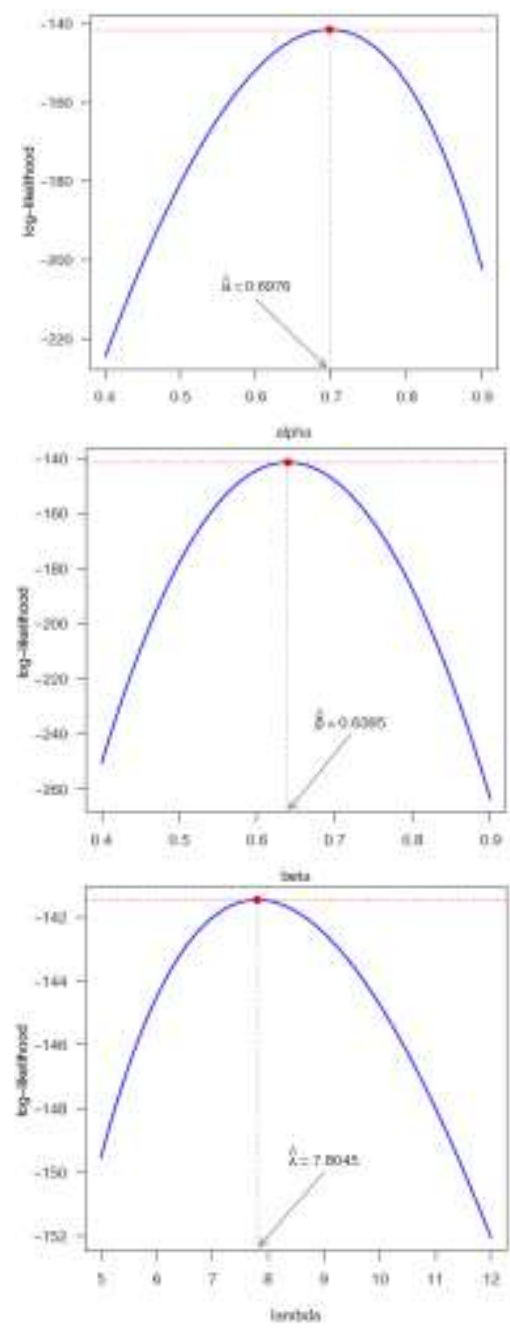

Figure 2. Graph of Profile log-likelihood function for the parameters $\alpha, \beta$ and $\lambda$ of PEP distribution.

The maximum likelihood estimates are calculated directly by using optim() function in $\mathrm{R}$ software ( $\mathrm{R}$ Core Team, 2020) and (Rizzo, 2008) by maximizing the likelihood 
function (3.1). We have obtained $\hat{\alpha}=0.6976, \hat{\beta}=0.6395$, $\hat{\lambda}=7.8045$ and corresponding Log-Likelihood value is 141.4625. In Table 1 we have demonstrated the MLE's with their standard errors (SE) and 95\% confidence interval for $\alpha$, $\beta$, and $\lambda$.

Table 1. MLE, SE and 95\% confidence interval

\begin{tabular}{llll}
\hline Parameter & MLE & SE & 95\% ACI \\
\hline alpha & 0.6976 & 0.1663 & $(0.3717,1.0235)$ \\
beta & 0.6395 & 0.1717 & $(0.3030,0.9760)$ \\
lambda & 7.8045 & 3.3750 & $(1.1895,14.4195)$ \\
\hline
\end{tabular}

Hence the Hessian variance-covariance matrix is obtained as,

$\left[-\left.H(\underline{\tau})\right|_{(\underline{\tau}=\underline{\hat{\tau}})}\right]^{-1}=\left(\begin{array}{ccc}0.02765481 & -0.02828359 & -0.5288098 \\ -0.02828359 & 0.02947504 & 0.5595579 \\ -0.52880981 & 0.55955793 & 11.3908809\end{array}\right)$

The Q-Q plots and P-P plot of PEP distribution are displayed in Figure 3. It is observed that the distribution fits the data excellently.
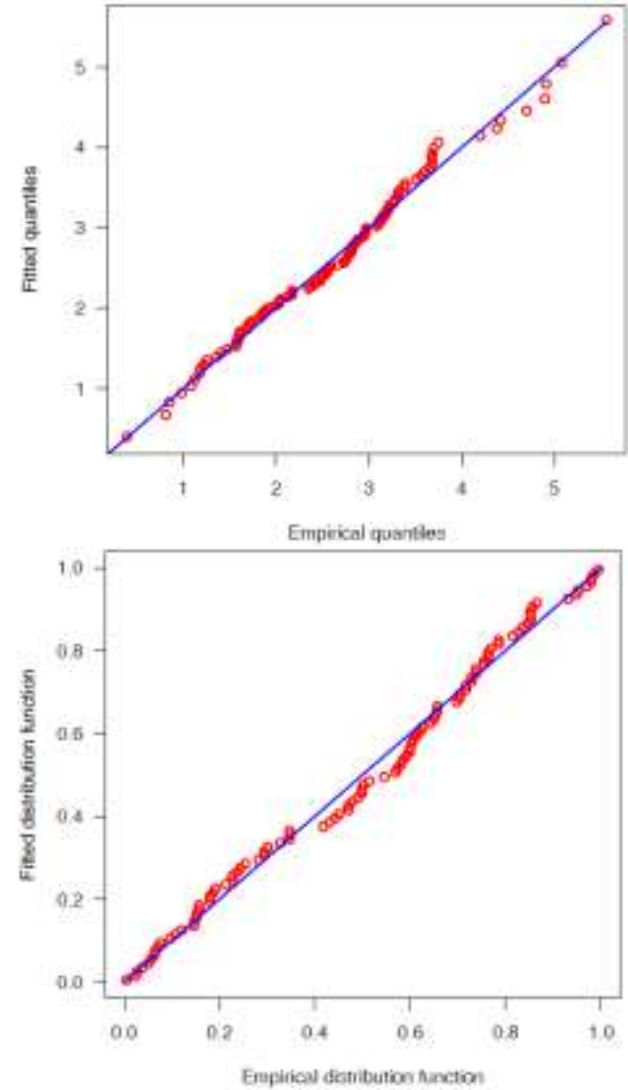

Figure 3. The Q-Q plot (upper panel) and P-P plot (lower panel) of PEP distribution
We have fitted the PEP distribution and some selected distributions which are as follows,

\section{A. Weibull extension (WE) distribution}

The probability density function of Weibull extension (WE) distribution (Tang et al., 2003) with three parameters $(\alpha, \beta, \lambda)$ is

$f_{W E}(x ; \alpha, \beta, \lambda)=\lambda \beta\left(\frac{x}{\alpha}\right)^{\beta-1} \exp \left(\frac{x}{\alpha}\right)^{\beta} \exp \left\{-\lambda \alpha\left(\exp \left(\frac{x}{\alpha}\right)^{\beta}-1\right)\right\} ; x>0$

$\alpha>0, \beta>0$ and $\lambda>0$

\section{B. Weighted Lindley distribution:}

Ghitany et al. (2011) has introduced a two-parameter weighted Lindley distribution and its PDF can be written as,

$f_{W L}(x)=\frac{\theta^{\alpha+1}}{(\alpha+\theta) \Gamma(\alpha)} x^{\alpha-1}(1+x) e^{-\theta x} \quad ; x \geq 0, \alpha>0, \theta>0$.

\section{Poisson-exponential distribution (PE)}

The probability density function of Poisson-exponential distribution was defined by (Louzada-Neto et al., 2011) also it was used by (Rodrigues et al., 2018) is

$$
f(x)=\frac{\beta \lambda}{\left(1-e^{-\lambda}\right)} e^{-\beta x} \exp \left(-\lambda e^{-\beta x}\right) ; \beta>0, \lambda>0, x>0
$$

\section{Exponential power $(\mathrm{EP})$ distribution:}

The probability density function Exponential power (EP) distribution (Smith \& Bain, 1975) is

$$
f_{E P}(x)=\alpha \lambda^{\alpha} x^{\alpha-1} e^{(\lambda x)^{\alpha}} \exp \left\{1-e^{(\lambda x)^{\alpha}}\right\} ;(\alpha, \lambda)>0, \quad x \geq 0 .
$$

where $\alpha$ and $\lambda$ are the shape and scale parameters respectively.

\section{E. Generalized Exponential (GE) distribution:}

The probability density function of generalized exponential distribution (Gupta \& Kundu, 1999) is.

$$
f_{G E}(x ; \alpha, \lambda)=\alpha \lambda e^{-\lambda x}\left\{1-e^{-\lambda x}\right\}^{\alpha-1} ;(\alpha, \lambda)>0, x>0 \text {. }
$$

The negative log-likelihood value and the value of AIC, BIC, CAIC and HQIC are presented in Table 2. We conclude that the proposed model produces a better fit to the data taken than other models.

Table 2. Log-likelihood, AIC, BIC, CAIC and HQIC

\begin{tabular}{clllll}
\hline Model & -LL & AIC & BIC & CAIC & HQIC \\
\hline PEE & 141.4625 & 288.9251 & 296.7406 & 289.1751 & 292.0882 \\
WE & 141.5577 & 289.1153 & 296.9309 & 289.3653 & 292.2784 \\
\hline
\end{tabular}




\begin{tabular}{llllll}
\hline WL & 143.0426 & 290.0852 & 295.2955 & 290.2089 & 292.1939 \\
PE & 144.2051 & 292.4102 & 297.6205 & 292.5339 & 294.5189 \\
EP & 145.9589 & 295.9179 & 301.1282 & 296.0391 & 298.0266 \\
GE & 146.1823 & 296.3646 & 301.5749 & 296.4883 & 298.4733 \\
\hline
\end{tabular}

The histogram and the fitted density functions are displayed in Figure 4 which compares the distribution function for the different models with the empirical distribution function that produces the same. Therefore, the given data sets illustrate the proposed distribution gets better fit and more reliable results from other alternatives.
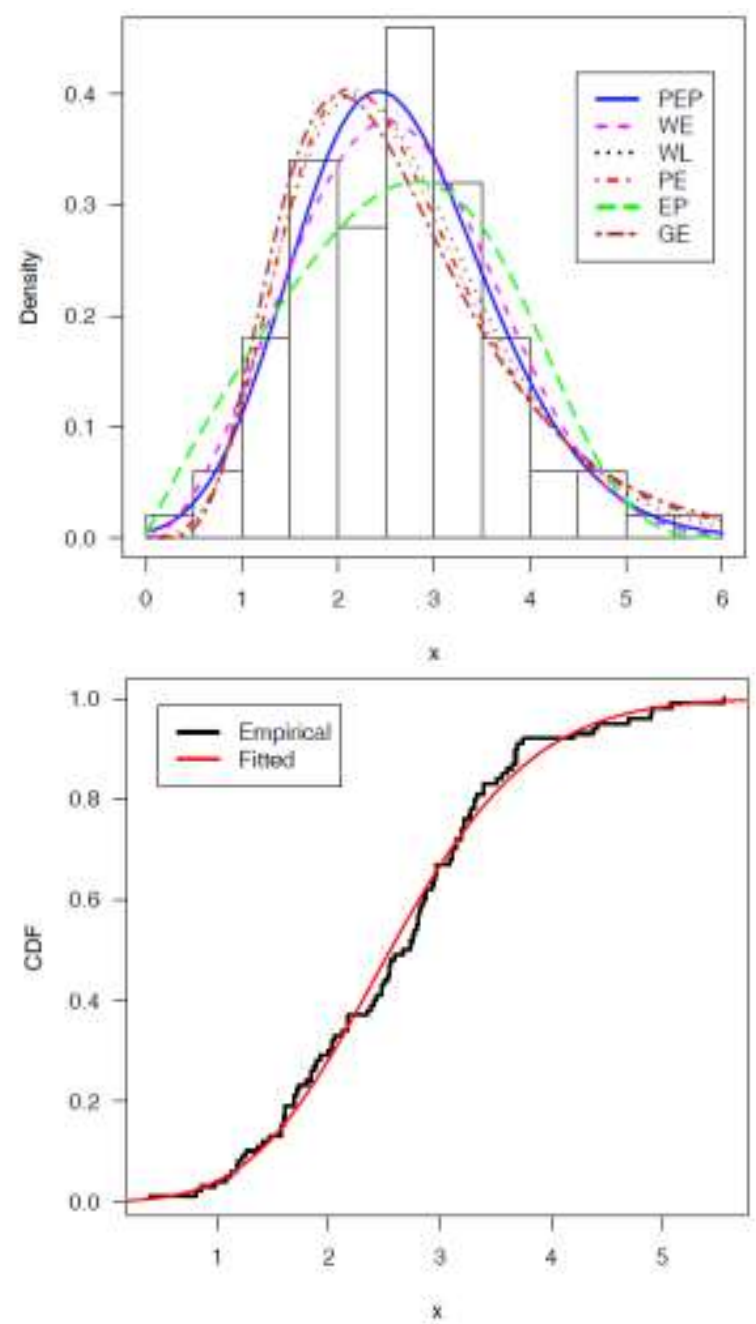

Figure 4. The Histogram and the PDF of fitted distributions (upper panel) and Empirical CDF with estimated CDF (lower panel).

In Table 3 we have displayed the value of the test statistics the Kolmogorov-Simnorov (KS), the Anderson-Darling (AD) and the Cramer-Von Mises (CVM) statistics and their corresponding $p$-value of different models. The result verifies that the proposed model has the minimum value of the test statistic and higher $p$-value hence we conclude that the Poisson exponential power distribution is better in the view of goodness-of-fit.
Table 3. The goodness-of-fit statistics and their corresponding p-value

\begin{tabular}{clll}
\hline Model & KS $(\boldsymbol{p}$-value $)$ & $\boldsymbol{A D}(\boldsymbol{p}$-value $)$ & $\boldsymbol{C V M}(\boldsymbol{p}$-value $)$ \\
\hline PEE & $0.0688(0.7309)$ & $0.0749(0.7232)$ & $0.4199(0.8284)$ \\
WE & $0.0607(0.8542)$ & $0.0635(0.7932)$ & $0.4212(0.8268)$ \\
WL & $0.0922(0.3637)$ & $0.1449(0.4061)$ & $0.7314(0.5328)$ \\
PE & $0.0954(0.3229)$ & $0.1724(0.3284)$ & $0.9157(0.4044)$ \\
EP & $0.0993(0.2771)$ & $0.1861(0.2963)$ & $1.3081(0.2297)$ \\
GE & $0.1078(0.1959)$ & $0.2293(0.2174)$ & $1.2250(0.2581)$ \\
\hline
\end{tabular}

\section{CONCLUSION}

In this study, we have presented a new expansion of the exponential power model called Poisson exponential power (PEP) distribution. Some statistical and mathematical properties of the PEP model have been discussed. From the graphical analysis of PDF and HRF, the proposed model is versatile and increasing, decreasing and upside bathtub hazard function. We have calculated the maximum likelihood estimates of the model parameters and the corresponding confidence intervals and information matrix of the MLE's. We have also illustrated the application of PEP distribution using a real data set and found quite useful and behaves better in terms of fitting as compared to some selected models. It may be an alternative model for practitioners in the area of theory and applied statistics.

\section{REFERENCES}

1. Adamidis, K., Loukas, S., 1998. A lifetime distribution with decreasing failure rate. Statistics and Probability Letters, 39, 35-42.

2. Alkarni, S. and Oraby, A. (2012). A compound class of Poisson and lifetime distributions, J. Stat. Appl. Pro., 1(1), 45-51.

3. Bader, M. G., \& Priest, A. M. (1982). Statistical aspects of fiber and bundle strength in hybrid composites. Progress in science and engineering of composites, 1129-1136.

4. Barreto-Souza, W. and Cribari-Neto, F. (2009). A generalization of the exponential-Poisson distribution. Statistics and Probability Letters, 79, 2493-2500.

5. Cancho, V. G., Louzada-Neto, F. and Barriga, G. D. C. (2011). The Poisson-exponential lifetime distribution. Computational Statistics and Data Analysis, 55, 677-686.

6. Ghitany, M. E., Atieh, B., \& Nadarajah, S. (2008). Lindley distribution and its application. 
Mathematics and computers in simulation, 78(4), 493-506.

7. Ghitany, M. E., Alqallaf, F., Al-Mutairi, D. K., \& Husain, H. A. (2011). A two-parameter weighted Lindley distribution and its applications to survival data. Mathematics and Computers in simulation, 81(6), 1190-1201.

8. Ghitany, M. E., Al-Mutairi, D. K., and Aboukhamseen, S. M., (2013). Estimation of the reliability of a stress-strength system from power Lindley distributions, Communications in Statistics - Simulation and Computation, 78, 493-506.

9. Gupta, R. D., \& Kundu, D. (1999). Theory \& methods: Generalized exponential distributions. Australian \& New Zealand Journal of Statistics, 41(2), 173-188.

10. Hyndman. R.J. \& Fan, Y. (1996). Sample quantiles in statistical packages. The American Statistician, 50(4): 361-365.

11. Joshi, R. M. (2015). An extension of exponential distribution: Theory and Applications. J. Nat. Acad. Math, 29, 99-108.

12. Joshi, R. K. \& Kumar, V. (2020). Lindley exponential power distribution with Properties and Applications. International Journal for Research in Applied Science \& Engineering Technology (IJRASET), 8(10), 22-30.

13. Kaviayarasu, V. \& Fawaz, P. (2017). A Reliability Sampling Plan to ensure Percentiles through Weibull Poisson Distribution, International Journal of Pure and Applied Mathematics, 117(13), 155-163.

14. Kenney, J. F. \& Keeping. (1962). E. S. Mathematics of Statistics. ( $3^{\text {rd }}$ ed.). Chapman \& Hall Ltd, New Jersey.

15. Kus, C. (2007). A new lifetime distribution. Computational Statistics and Data Analysis 51, 4497-4509.

16. Kyurkchiev, V. E. S. S. E. L. I. N., Kiskinov, H. R. I. S. T. O., Rahneva, O. L. G. A., \& Spasov, G. E. O. R. G. I. (2018). A Note on the Exponentiated Exponential-Poisson Software Reliability Model. Neural, Parallel, and Scientific Computations, 26(3), 257-267.

17. Louzada-Neto, F., Cancho, V.G. \& Barriga, G.D.C. (2011). The Poisson-exponential distribution: a Bayesian approach, Journal of Applied Statistics, 38:6, 1239-1248.

18. Louzada, F., Luiz Ramos, P., \& Henrique Ferreira, P. (2020). Exponential-Poisson distribution: estimation and applications to rainfall and aircraft data with zero occurrences. Communications in Statistics-Simulation and Computation, 49(4), 1024-1043.
19. Lu, W. \& Shi, D. (2012). A new compounding life distribution: the Weibull-Poisson distribution, Journal of Applied Statistics, 39:1, 21-38.

20. Percontini, A., Blas, B., \& Cordeiro, G. M. (2013). The beta weibull poisson distribution. Chilean journal of Statistics, 4(2), 3-26.

21. Mahmoudi, E., \& Sepahdar, A. (2013). Exponentiated Weibull-Poisson distribution: Model, properties and applications. Mathematics and computers in simulation, 92, 76-97.

22. Moors, J. J. A. (1988). A quantile alternative for kurtosis. Journal of the Royal Statistical Society: Series D (The Statistician), 37(1), 25-32.

23. Morais, A. \& Barreto-Souza,W., (2011). A compound class of Weibull and power series distributions. Computational Statistics and Data Analysis, 55, 1410-1425.

24. Nadarajah, S., \& Kotz, S. (2006). The beta exponential distribution. Reliability engineering \& system safety, 91(6), 689-697.

25. Nichols, M. D., \& Padgett, W. J. (2006). A bootstrap control chart for Weibull percentiles. Quality and reliability engineering international, 22(2), 141-151.

26. R Core Team (2020). R: A language and environment for statistical computing. $\mathrm{R}$ Foundation for Statistical Computing, Vienna, Austria. URL https://www.R-project.org/.

27. Ristić, M. M., \& Nadarajah, S. (2014). A new lifetime distribution. Journal of Statistical Computation and Simulation, 84(1), 135-150.

28. Rizzo, M. L. (2008). Statistical computing with $R$. Chapman \& Hall/CRC.

29. Rodrigues, G.C., Louzada, F. \& Ramos, P.L. (2018). Poisson-exponential distribution: different methods of estimation, Journal of Applied Statistics, 45(1), 128-144.

30. Smith, R.M. and Bain, L.J. (1975). An exponential power life-test distribution, Communications in Statistics, 4, 469-481.

31. Srivastava, A. K., \& Kumar, V. (2011). Analysis of software reliability data using exponential power model. IJACSA Editorial.

32. Tang, Y., Xie, M., \& Goh, T. N. (2003). Statistical analysis of a Weibull extension model. Communications in Statistics-Theory and Methods, 32(5), 913-928. 